\title{
Chemistry Nobel Prize 2002 Goes to Analytical Chemistry
}

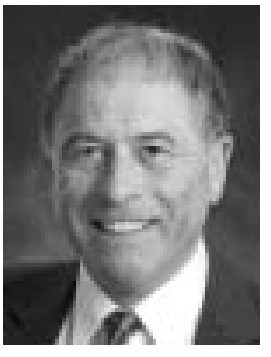

K. Wüthrich

October 2002 was a great month for Swiss science with Kurt Wüthrich of the ETH Zürich winning the Chemistry Nobel prize 2002. The other half of the 2002 Chemistry Nobel prize went jointly to John B. Fenn of the Virginia Commonwealth University (Richmond, USA) and to Koichi Tanaka of Shimadzu Corp. (Kyoto, Japan), who independently developed techniques to ionize large molecules for study by mass spectrometry. This recognition for the development of analytical methods for high molecular weight molecules is well deserved and even overdue, given the enormous impact these methodologies have had in biochemistry, proteomics, polymer science, and many other fields.

Kurt Wüthrich's achievements in NMR spectroscopy of macromolecules have already been honored with a special article in this journal (Chimia 2002, 56, 712-713). This article is thus dedicated to the mass spectrometric part of the Chemistry Nobel 2002. Fenn and Tanaka were chosen by the Royal Swedish Academy of Sciences for 'their development of soft desorption methods for mass spectrometric analyses of biological macromolecules'.

Fenn, 85, won for the development of electrospray ionization (ESI), which gives researchers a rapid and easy-to-implement method for analyzing a wide range of polar molecules, such as proteins, carbohydrates, and nucleic acids. ESI employs nebulization and charging of a liquid sample stream that is passed through a capillary by the application of a high, inhomogeneous electric field. Nowadays, researchers are able to ionize huge molecules, for example protein

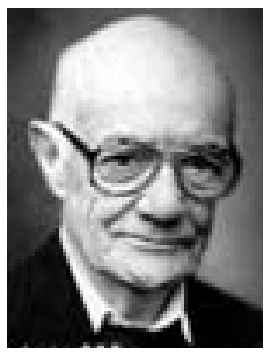

J.B. Fenn

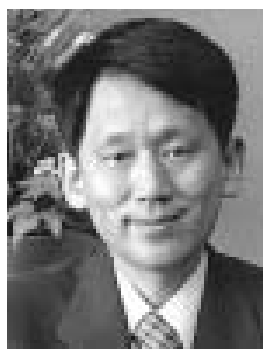

K. Tanaka complexes, the ribosome, or even intact viruses by using ESI. Fenn did his original work on ESI while a professor at Yale University in the early 1980s. Coming from the field of molecular beams, he was following up on earlier (but unsuccessful) work by Malcolm Dole to produce gas-phase ions from very large molecules. Fenn's experience with molecular beam methods helped him to succeed where the earlier research in this direction had failed. Because electrospray ionization produces multiply charged ions, it was relatively easy to observe macromolecular ions even in the early days of ESI: due to fairly low $\mathrm{m} / \mathrm{z}$ values, most signals were observable with standard quadrupole mass spectrometers. Initially Fenn had trouble getting acceptance from other mass spectrometrists, who did not pay much attention to the potential use of ESI: when Fenn presented his initial results at an American Chemical Society meeting in 1984 , only a handful of people attended the session. However, the opportunity to use ESI for interfacing liquid separation methods with mass spectrometry quickly convinced many researchers as well as instrument manufacturers of the potential of this method. It is now the most widely used method for LC-MS interfacing.

Tanaka, 43, won for his work on laser desorption/ionization of macromolecules, work he did in his late twenties in Japan. Tanaka, one of the youngest chemistry laureates, has been an R\&D engineer with Shimadzu throughout his career and has never earned a doctorate. He first presented his results of laser desorption/ionization of high molecular weight molecules in 1987 at the 2nd Japan-China Joint Symposium on Mass Spectrometry, and published them in 1988 (Rapid Commun. Mass Spectrom. 1988, 2, 151-153). In his original work, Tanaka and his coworkers used a sample preparation where the analyte is mixed with ultrafine cobalt powder and glycerol as a vacuum-stable binding medium. When irradiated with a pulse from a low energy nitrogen laser, the metal particles heat up rapidly, releasing glycerol and intact analyte molecules into the gas phase. A small proportion of them forms ions during the desorption/ionization event that are then analyzed by a time-of-flight mass spectrometer. The method is hardly used in its original form nowadays but became enormously popular by the development of a closely related method, matrix-assisted laser desorption/ionization (MALDI). Developed by Franz Hillenkamp and Michael Karas, then at the University of Münster, Germany, MALDI is now the method of choice for proteomics as well as for the mass spectrometric characterization of synthetic polymers.

\section{Renato Zenobi}

Laboratorium für Organische Chemie ETH Hönggerberg HCI

CH-8093 Zürich 